
2 Research Square
Preprints are preliminary reports that have not undergone peer review.
They should not be considered conclusive, used to inform clinical practice, or referenced by the media as validated information.

\title{
Radiomics analysis of magnetic resonance imaging helps to identify preclinical Alzheimer's disease: an exploratory study
}

Tao-Ran Li

Xuanwu Hospital

Yue Wu

Shanghai University

Juan-Juan Jiang

Shanghai University

Jie-Hui Jiang

Shanghai University

Ying Han ( $\nabla$ hanying@xwh.ccmu.edu.cn )

Xuanwu Hospital

\section{Research}

Keywords: Alzheimer's disease, Preclinical AD, Radiomics, MRI, fMRI, DTI, Features, Imaging biomarker, Cross-validation

Posted Date: July 30th, 2020

DOI: https://doi.org/10.21203/rs.3.rs-49621/v1

License: (c) (1) This work is licensed under a Creative Commons Attribution 4.0 International License. Read Full License 


\section{Abstract}

Background: Diagnosing Alzheimer's disease (AD) in the preclinical stage offers opportunities to early intervention, however, there is a lack of convenient biomarkers currently. By using the methods of radiomics analysis, we aimed to determine whether the features extracted from multi-parameter magnetic resonance imaging (MRI) can be used as potential biomarkers.

Methods: This study is part of the SILCODE project (NCT03370744). All participants were cognitively healthy at baseline. The cohort 1 ( $\mathrm{n}=183$ ) was divided into individuals with preclinical $A D(n=78)$ and controls $(n=105)$ by amyloid-positron emission tomography, used as the training dataset $(80 \%)$ and validation dataset (the rest $20 \%$ ); cohort $2(n=51)$ was divided into "converters" and "non-converters" by individuals' future cognitive status, used as a separate test dataset; cohort 3 included 37 "converters" (13 from ADNI), was used as another test set for independent longitudinal researches. We extracted radiomics features from multi-parameter MRI of each participant, used t-tests, autocorrelation tests, and three independent selection algorithms, respectively, to select features. Then we established two classification models (support vector machine (SVM) and random forest (RF)) to verify the efficiency of retained features. Five-fold cross-validation and 100 repetitions were carried out for the above process. Furthermore, the acquired stable high-frequency features were tested in cohort 3 by paired two-sample t-tests and survival analyses, in order to identify whether their levels change with cognitive decline and impact conversion time.

Results: The SVM and RF models both showed excellent classification efficiency, with the average accuracy of $89.65 \%-95.90 \%$ and $87.07 \%-90.81 \%$ respectively in the validation set, $81.86 \%-89.10 \%$ and $83.19 \%-83.68 \%$ respectively in the test set. Three stable high-frequency features were identified, namely Large zone high-gray-level emphasis feature of right posterior cingulate gyrus, Variance feature of left superior parietal gyrus and Coarseness feature of left posterior cingulate gyrus, all based on structural MRI modality; their levels were correlated with amyloid- $\beta$ deposition, played good roles in predicting future cognitive decline (AUCs 64.9\%-76.1\%). In addition, levels of the Variance feature at baseline timepoint decreased with cognitive decline, and can affect the conversion time $(p<0.05)$.

Conclusion: In this exploratory study, we show radiomics features of multi-parameter MRI can be potential biomarkers of preclinical AD.

\section{Background}

Alzheimer's disease (AD) is an evolving challenge, which represents the largest unmet medical need because it's epidemic, irreversible and incurable currently ${ }^{[1,2]}$. A series of disappointing large-scale clinical trials on symptomatic patients have raised clinical consensus that we should move our focus forward ${ }^{[3,4]}$. According to the latest National Institute on Ageing-Alzheimer's Association (NIA-AA) diagnostic framework, preclinical AD patients refer to the cognitively healthy individuals but accompanying brain amyloid- $\beta(A \beta)$ deposition ${ }^{[5]}$, they have a higher risk of developing prospective cognitive and functional decline ${ }^{[6,}$

7]. The accurate super-early diagnosis of preclinical stage exactly provides a window period for interventions, is of great clinical importance and being the first imperative.

Since $A \beta$ deposition is the gold standard for the diagnosis of preclinical $A D$, how to detect it has become a crucial issue. Currently, the international recognized state-of-the-art biopsy assessments of brain $A \beta$ depend on amyloid-positron emission tomography (PET) imaging and cerebrospinal fluid analysis ${ }^{[5]}$, however, their popularization is limited by costliness and invasiveness ${ }^{[8]}$. Hence, an urgent need for convenient and inexpensive technique still exists. Magnetic resonance imaging (MRI) has been widely used in the clinical evaluation of neurodegeneration, and was compiled into the $A D$ diagnostic framework ${ }^{[5]}$; comparatively, functional MRI (fMRI) and diffusion tensor imaging (DTI) are basically limited to scientific research. Considerable research developments have been acquired in the discrimination of mild cognitive impairment $(\mathrm{MCl})$ and dementia by using these different modal images ${ }^{[9]}$, which are promising in identifying preclinical $A D$, however, there is a lack of diagnostic biomarkers at this stage.

Radiomics, a method of high-dimensional minable data analysis, can quantitatively examine a large set of phenotypic features and has previously been

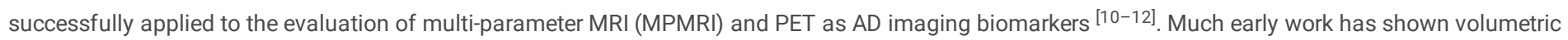
and morphometric indices of specific brain regions, including hippocampus, thalamus, callosum and cingulate gyrus, have already changed in the prophase of cognitive decline ${ }^{[13-17]}$. Recent progresses of texture analysis suggested the abnormalities of textural features occur earlier ${ }^{[18-20]}$, can also distinguish among healthy controls, AD-MCl and AD-dementia patients based on cortices, subcortical regions or whole-brain analysis ${ }^{[19,21-27]}$, and their accuracy in predicting $\mathrm{MCl}$ to dementia is higher than that of volume reduction ${ }^{[18,20,24]}$. Relative to controls, both AD-MCl and AD-dementia patients showed widespread changes of multiple indices of DTI ${ }^{[28,29]}$. These findings point to the possible use of radiomics analysis of MPMRI as a measure of neurodegenerative process in $A D$, which may contain unique information about changes at the microscopic level that can occur before changes at the macroscopic level, such as atrophy. However, to the best of our knowledge, there is no such studies focus on preclinical AD.

With this study we aimed to (a) explore novel imaging biomarkers based on radiomics analysis of MPMRI (structural MRI (sMRI), fMRI, DTI); (b) employ classification models to discriminate preclinical $A D$ based on radiomics features.

\section{Methods}

\subsection{Study design}

The comprehensive workflow was shown in Figure 1, including cohorts' establishment (A), preprocessing of images (B), extraction and selection of radiomics features (C), models' establishment, classification experiments, correlation analysis (D), and longitudinal studies of typical features (E).

\subsection{Participants}


The study was part of the Sino Longitudinal Study on Cognitive Decline (SILCODE), an ongoing prospective cohort study (ClinicalTrials.gov identifier: NCT03370744; protocol can be accessed at ClinicalTrials.gov) ${ }^{[30]}$, which centers on Xuanwu Hospital, and the alliance includes 94 hospitals from 50 cities in China. The SILCODE project is a constellation of interconnected sub-studies, and one of its aims is to assess the diagnostic application of imaging in different stages of cognitive continuum. Therefore, baseline standardized clinical evaluation and MPMRI were offered to all participants, resulting in the enrollment of 1594 with different diagnoses, ranging from cognitively unimpaired to dementia. In this study, we established three cohorts from the database and Alzheimer's Disease Neuroimaging Initiative (ADNI, www.loni.ucla.edu/ADNI) with high selectivity. In cohort 1, one hundred and eighty-three cognitively healthy participants with amyloid-PET imaging were being included between July 2016 and November 2018 sequentially, they were all from SILCODE project (Supplementary Figure 1A). In cohort 2, fifty-one participants were included, they participated in SILCODE project from December 2009 to December 2015 and were selected retrospectively; they were interviewed every 10-15 months until the end of 2019, with 24 had future cognitive decline and 27 remained healthy (Supplementary Figure 1B). In cohort 3, the 24 "converters" from cohort 2 and additional 13 individuals from ADNI were included, they all underwent MPMRI examinations at baseline timepoint and when they were first identified cognitive deterioration. The entry criteria of healthy individuals referred to our previous references ${ }^{[30,31]}$; the diagnosis of dementia referred to the guidelines of NIA-AA workgroups ${ }^{[32]}$; MCI was based on the Petersen's criteria (before 2016) ${ }^{[33]}$ or a neuropsychological method (after 2016) ${ }^{[34]}$.

Participants in cohort 1 all underwent a dynamic scan with Florbetapir F-18 (AV45), the whole brain voxel-wise standardized uptake value ratio (SUVR) was normalized to the whole cerebellum, representing the mean cortical SUVR. For the dichotomy, amyloid-PET positivity, that is participants who in the preclinical stage of Alzheimer's continuum, was defined a priori with the established cutoffs of $>1.18^{[35]}$. The result of each participant was confirmed by two senior radiologists.

Informed consent was obtained from all participants. Further details regarding the rigorous evaluation of our participants were presented in the Supplementary Figure 1 and Material.

\subsection{Imaging acquisition and preprocessing}

The MRI data of participants from SILCODE project were acquired using a 3.0-T MR imager (Magnetom Sonata; Siemens, Germany) before the year of 2016, or an integrated simultaneous 3.0-T TOF PET/MR (SIGNA; GE, USA) after that timepoint. Before undergoing imaging, subjects were instructed to keep their eyes closed but not fall asleep, to relax their minds, and to move as little as possible during imaging, and foam pads and headphones were used to minimize head movement and imager noise. The sMRI was obtained with a magnetization prepared rapid gradient echo sequence (Siemens/GE): repetition time (TR) $=1900$ $\mathrm{ms} / 6.9 \mathrm{~ms}$, echo time $(\mathrm{TE})=2.2 \mathrm{~ms} / 2.98 \mathrm{~ms}$, slice number = 176/192; fMRI was obtained with a single-shot gradient-echo echo planar imaging (EPI) sequence (Siemens/GE): TR = $2000 \mathrm{~ms} / 2000 \mathrm{~ms}, \mathrm{TE}=40 \mathrm{~ms} / 30 \mathrm{~ms}$, slice number $=28 / 28$; and a single-shot spin-echo diffusion-weighted EPI sequence was used for the DTI data (Siemens/GE): TR $=11000 \mathrm{~ms} / 16500 \mathrm{~ms}$, TE $=98 \mathrm{~ms} / 95.6 \mathrm{~ms}$, slice number $=60 / 75$. The detailed protocols could be found in the Supplementary Material.

The images of the ADNI participants were downloaded from the ADNI database, detailed information regarding the acquisition protocol is publicly available on the LONI website (https://ida.loni.usc.edu/login.jsp).

The standardized preprocessing of amyloid-PET and MPMRI referred to previous studies ${ }^{[30,36,37]}$. The original DICOM data were converted to NIfTI file format by using DCM2NII (https://people.cas.sc.edu/rorden/mricron/dcm2nii.html), then we processed the MPMRI and amyloid-PET separately for each participant. For sMRI, the images were normalized and showed a spatial resolution of $91 \times 109 \times 91$ with $2 \times 2 \times 2 \mathrm{~mm}^{3}$ voxel size after being segmented into gray matter, white matter and cerebrospinal fluid tissues, afterwards, we smoothed them by using an isotropic Gaussian smoothing kernel with the full-width at half maximum of $4 \times 4 \times 4 \mathrm{~mm}^{3}$. For fMRI, the first 10 time-point images were discarded for magnetization balance, after that, the remaining 230 time-point images were corrected and aligned to the first time-point image to correct head movements; the resultant motion-corrected volumes were co-registered to the anatomical T1-weighted images and normalized to the Montreal Neurological Institute template, resampling to a $3 \mathrm{~mm}$ cube voxel resolution. For DTI, we employed the Eddy Correct tool to correct the head motion and eddy current distortions ${ }^{[38,39]}$, and used the Brain Extraction tool to remove the non-brain tissues of the BO image and create the brain mask ${ }^{[40]}$, then we adopted the DTIFIT tool to fit the diffusion tensor at each voxel and produced four parameter maps, encompassing fractional anisotropy, mean diffusivity, axial diffusivity and radial diffusivity ${ }^{[41]}$. For amyloid-PET, the structural images were individually registered to the averaged PET images, then we performed segmentation of all the co-registered structural images, spatially normalized the PET images to the Montreal Neurological Institute standard space by using the forward parameters (estimated during the segmentation), smoothed the images with an $8 \mathrm{~mm}$ full width at half maximum Gaussian kernel.

The sMRI and fMRI images were preprocessed by using the Data Processing Assistant for Resting-state fMRI (DPASF; http://www.rfmri.org/DPARSF) implemented in the MATLAB R2018a (Mathworks, Natick, MA, USA) ${ }^{[42]}$; DTI images were by the pipeline for analyzing brain diffusion images (PANDA) implemented based on the FMRIB Software Library (FSL) ${ }^{[43]}$; amyloid-PET data were by the Statistical Parametric Mapping (SPM12; https://www.fil.ion.ucl.ac.uk/spm/software/spm12) implemented in the MATLAB.

\subsection{Features extraction}

The features extraction was performed for each modality separately. For SMRI, 43 texture features and 172 wavelet features of each region of interest (ROI) (116 in total, based on the AAL template) were extracted; for fMRI, 43 texture features of each ROI were extracted; for DTI, we calculated the white matter tracts 
and viewed the fractional anisotropy, mean diffusivity, axial diffusivity and radial diffusivity as features. All these extracted features were adjusted by using linear regression to control the impacts of age, gender and education before selection.

Features extraction of SMRI and fMRI was performed on the Texture Toolbox from radiomics tools developed by Vallieres et al. (https://github.com/mvallieres/radiomics), based on MATLAB [44]; for DTI, the procedure was carried out on the PANDA ${ }^{[43,45]}$. More details were described in the Supplementary Table 1 and Material.

\subsection{Features dimensionality reduction and selection}

This step was achieved on MATLAB. More specifically, we performed a 5 -fold cross-validation on the dataset of cohort 1 , that is, the data was randomly divided into a training set (80\%) and a validation set (the rest $20 \%$ ); in the training set, three steps including t-tests, autocorrelation tests and independent three different algorithms (Fisher score, Least absolute shrinkage and selection operator (Lasso) and Max-Relevance and Min-Redundancy (mRMR)) were adopted to filter the redundant and meaningless features; the remaining features were retained and incorporated into classification models. Importantly, we repeated the above steps 100 times. More details could be found in the Supplementary Material.

In addition, for each type of above three algorithms, we calculated the number of occurrences of each retained feature, ranging from 0 to 500 . The top ten most frequently appeared features were defined as high-frequency features, and the stable high-frequency features means the overlaps among the three perturbations.

\subsection{Classification experiments}

We established two classification models, support vector machine (SVM) and random forest (RF), to verify the performance of retained features in the validation ( $20 \%$ of cohort 1$)$ and test set (cohort 2 ) respectively. The SVM model employed three different kernels, sigmoid, linear and radial basis.

Corresponding to the retained features, there were 500 permutation experiments under the algorithm of Fisher score, Lasso or mRMR, then the final results of accuracy, sensitivity and specificity were presented as average values \pm standard deviation (SD) of each model.

In addition, the receiver operating characteristic (ROC) analyses were performed to evaluate the ability of each stable high-frequency feature in predicting prospective cognitive decline of participants in the test set, and the areas under curve (AUCs) were calculated. The analysis was achieved by SPSS 13.0 software (Chicago, IL, USA).

Details could be found in the Supplementary Material.

\subsection{Longitudinal analyses}

As an independent longitudinal research, this study aimed to further verify the role of stable high-frequency features identified from the training dataset (80\% of cohort 1) in another separate test dataset, that is the cohort 3 . The features extraction was identical to above-mentioned. In order to test whether the levels of stable high-frequency features change with cognitive decline, we made comparisons at the two different timepoints of cognitive stages, and in order to verify whether these features have impacts on the conversion times of individuals, we performed the survival analyses.

\subsection{Statistical analysis}

The demographic data of participants were summarized as numbers (\%) or mean \pm SD for categorical and continuous variables, respectively. The betweengroup comparisons were performed by using chi-square test for categorical variables, or the two-sample t-test for continuous variables (two-tailed). A $p<0.05$ was considered significant.

In the process of dimensionality reduction, the two-sample t-test was two-tailed and considered significant when $p<0.05$; for the autocorrelation test, we calculated the Pearson correlation coefficients between features and considered the paired features had a high correlation when values in the pair-wise correlation were greater than 0.8 . Furthermore, in order to better understand the association between radiomics features and iconic pathological changes of $A D$, we made Pearson correlations to evaluate the relationship between stable high-frequency features and mean cortical SUVR values, and acquired the results after adjusting for age, gender, education, and Montreal cognitive assessment (MoCA) scores.

In the longitudinal analyses, we drew the changing trajectory of each stable high-frequency feature at the individual level, and performed the paired twosample t-tests at the group level (two-tailed, $p<0.05$ ). In the survival analyses, individuals of cohort 3 were equally divided into two parts, the high-level group (n $=18$ ) and low-level group ( $n=19)$, according to the median level of each stable high-frequency feature, respectively, afterwards, cumulative probabilities of clinical conversion by the two groups were displayed according to the Kaplan-Meier method, then the survival curves were compared between groups in an univariate analysis applying the log-rank test.

These above analyses were performed in SPSS or MATLAB.

\section{Results}

\subsection{Participants}


In cohort 1, 183 healthy participants were included. Their clinical and MPMRI examinations were almost continuous, and the amyloid-PET was performed within 3 months of the MPMRI scan. Eventually, seventy-eight amyloid-positive and 105 negative participants were identified. Compared to the negative individuals, individuals who were positive were older $(p=0.039)$ and had higher AV45 SUVR $(p<0.0001)$, while there were no statistical differences of other clinical data (Table 1).

In cohort 2, additional 51 healthy participants were dichotomized due to their future cognitive outcomes. They were interviewed every $10-15$ months and we found the cognition of 24 deteriorated after an average of 41.2 months (IQR: $24.5-52.7$ ), with 23 progressed to $\mathrm{MCl}$ and 1 to dementia, while the remaining remained healthy after at least 3 follow-up visits (54.8 months, IQR: 48.9-58.9). As shown in Table 1, there were no differences between the two groups.

Cohort 3 included the 24 "converters" from cohort 2 and 13 from ADNI, their average score of MoCA scale slumped from $23.7 \pm 2.7$ at baseline timepoint to $20.5 \pm 3.8$ at follow-up timepoint. The average conversion time of ADNI participants was 62.1 months (IQR: $55.1-66.5$ ), of total individuals was 48.1 months (IQR: 27.8-61.7). The individuals from ADNI were used as an additional supplement, with 12 progressed from cognitively healthy to $\mathrm{MCl}$ and 1 to dementia. Other data could be acquired in Table 1.

\subsection{Features extraction, dimensionality reduction and selection}

For each participant of the three cohorts, 30128 features were extracted, including 24940 features from sMRI, 4988 from fMRI and 200 from DTI. To avoid over-fitting, these features were screened before being included into classification models. In the training set, 9,000-11,000 features were retained after the two-sample t-tests $(p<0.05)$ and 2200-2500 types of uncorrelated features were reserved after the autocorrelation tests. The remaining features were further filtered by three independent selection algorithms respectively, more specifically, we retained the top 50 ranked features by using the Fisher score test, $50-70$ features using the Lasso method, and top 50 ranked features after the mRMR test.

Generally, the retained features showed consistency in repeated experiments. As shown in Table 2, there were 10 high-frequency features of each composite function disturbance, notably, they were all based on sMRI modality. For the features selected from the disturbance containing Fisher score test, the frequency was 420-500 times, mainly originated from the posterior cingulate (left, 3/10; right, 3/10); for the features selected from Lasso, the frequency was 383-468 and no specific regions were identified; for the features selected from mRMR, the frequency was 320-495, also mainly from the posterior cingulate (left, 4/10; right, 2/10).

Three stable high-frequency features were identified during the process, they were undisturbed by the combined disturbances, and may of great importance in the preclinical stage of AD, specifically as follows: the Large zone high-gray-level emphasis (LZHGE) feature of right posterior cingulate gyrus on sMRI (ID: 11517) (459 times on average), the Variance feature of left superior parietal gyrus on sMRI (ID: 27442) (450 times on average) and the Coarseness feature of left posterior cingulate gyrus on SMRI (ID: 6489) (387 times on average). Additionally, among features with the number of occurrences greater than 300 , another two were also identified as overlaps, the LZHGE feature of left posterior cingulate gyrus on sMRI (ID: 6486) (458 times on average) and the Zone-size variance feature of right cerebellum-crus2 on SMRI (ID: 28977) (319 times on average).

Other retained features that occurred more than 300 times and the meanings of stable high-frequency features were described in the Supplementary Table 2 and Material.

\subsection{Classification experiments}

We introduced two types of models to determine whether the retained features were compatible for classification analysis. The Table 3 presented the classifier performance results in terms of accuracy, sensibility and specificity. As shown, the SVM model (radial basis kernel) showed excellent classification efficiency, with the average accuracy up to $90.23 \%-95.90 \%$ (sensibility, $85.91 \%-92.82 \%$; specificity, $93.71 \%-98.26 \%$ ) in the validation set, and $84.48 \%-88.94 \%$ (sensibility, $79.76 \%-82.87 \%$; specificity, $85.98 \%-96.70 \%$ ) in the test set. Similar results were acquired in the RF model (Table 3 ) or SVM models with the other two kernels (Supplementary Table 3). In contrast, the average accuracy of pure clinical data-based models in diagnosing preclinical AD reached only random level accuracy, was $55.93 \%-56.01 \%$ (details were presented in the Supplementary Table 4 and Material).

We further verified the classification efficiency of stable high-frequency features on the test set, and found their individual AUCs ranged from 0.649 to 0.761 , when we combined them together, the predictive ability was improved (AUCs $=0.839$ ) (Figure 2A-D). In addition, the feature 6486 also played a good classification effect (AUCs $=0.739$ ), and improved the AUCs to 0.863 when we combined it with the three stable features (Supplementary Figure 2 ). In contract, the performance of feature 28977 was too bad to draw a ROC curve. These results indicated that radiomics analysis was a reliable feature extraction method in the preclinical stage of $A D$, and provided promising imaging biomarkers in identifying cognitively healthy individuals with future cognitive decline.

\subsection{Correlation analysis}

In order to further understand the association between radiomics features and pathological changes of $A D$, we performed the correlation analysis between stable high-frequency features and mean cortical SUVR values of amyloid-PET and found they were highly correlated. In detail, the feature 6489 levels were positively correlated with SUVR values ( $r=0.433, p<0.0001$, Figure 2E), while the feature 11517 and 27442 levels were both inversely correlated with SUVR values $(r=-0.416, p<0.0001$, Figure $2 F ; r=-0.348, p<0.0001$, Figure $2 G)$. Similar results were found in the feature $6486(r=-0.400, p<0.0001)$

(Supplementary Figure 2). The correlation results did not change after adjusting for age, gender, education, and MoCA score (Supplementary Figure 3 ). Our

Page 5/14 
findings revealed that high correlations between levels of these features and A $\beta$ depositions, suggesting that radiomics features based on MPMRI may reflect pathological changes in the brain and be used for the diagnosis of $A D$.

\section{5. longitudinal analyses}

In this study, the 37 participants of cohort 3 were followed up until cognitive impairment was found. First, we detected their longitudinal changes of each stable high-frequency feature. As shown, the feature 6489 and 11517 did not show isotropic changes of the two cognitive stages at the individual level (Figure $3 A, B)$, correspondingly, there were also no statistical differences between the two paired groups (Figure 3D, E), similar results were obtained of the feature 6486 (Supplementary Figure 4A, B); although some individuals had a heterogeneous change pattern of the feature 27442 (Figure 3C), but its levels in the cognitive impairment stage was still lower than that in the cognitively healthy stage $(p=0.0403)$ (Figure $3 F)$. Second, we made survival analysis of these features respectively. In detail, the median baseline level of feature 6489, 11517 and 27442 was $0.0297356,17228.308,0.865647$ respectively; Figure 3G-I showed the probability of cognitive impairment by levels of features > and $\leq$ these cutoffs, notably, in the comparison between paired groups, only grouping by feature 27442 was meaningful (log rank $p=0.015$ ). The result of feature 6486 was also unsatisfactory when grouped by the median level of 48.967 (log rank $p=0.442$ ) (Supplementary Figure $4 \mathrm{C}$ ). These results indicated that the levels of feature 27442 decreased with cognitive decline, and the deterioration occurred earlier when the baseline level was less than 0.865647 , however, considering the limited sample size, the value is for reference only, and it is more accurate to state that the baseline level can affect the conversion time.

\section{Discussion}

The real pathophysiological process of $A D$ is thought to begin several decades before symptom onset, generally followed a rigid progress pattern that is $A \beta$ accumulation-neurofibrillary tangles-neuronal damage; neurons had already damaged to some extent when cognitive impairment occurred ${ }^{[1,8]}$. Radiomics analysis can extract high-dimensional features of MPMRI, may identify imaging patterns of preclinical stage that cannot be recognized by human readers, however, there is a paucity of published literatures assessing radiomics features of individuals in preclinical stage of $A D$ or with future cognitive decline. In this ongoing prospective cohort study, we adopted a novel composite method to select features from the training dataset, established classification models and verified them in the validation set and test set respectively; we found both models can distinguish whether individuals were in the preclinical stage or whether their future cognition will decline, with the accuracy more than $80 \%$. In addition, three stable high-frequency features were identified, they were independent of perturbations and correlated with A $\beta$ deposition, all can classify the test set accurately (AUCs $64.9 \%$ to $76.1 \%$ ). In the independent longitudinal analyses, we further verified that levels of the feature 27442 (Variance feature of left superior parietal gyrus on sMRI) decreased with cognitive decline and impacted individuals' conversion time. Together, these data showed that radiomics features of MPMRI were expected to be important imaging biomarkers in accessing patients with preclinical $A D$.

Our previous researches confirmed that cognitively normal high-risk individuals of AD have already appeared alterations of brain functional networks (fMRI), white matter networks (DTI) or some refined areas (sMRI) ${ }^{[46-49]}$, suggesting there may be more unmined data of MPMRI in the preclinical stage. As expected, the pure clinical data-based classification models were meaningless in this stage, and the traditional volumetric and functional indices are also not sensitive enough (details were presented in the Supplementary Table 5 and Material). Although it is generally believed that radiomics analysis is more sensitive, but current studies are still limited to symptomatic stages $[18,19,22,23,29]$. Chaddad et al. found the features derived from a single subcortical region produced an AUCs up to $80 \%$ for classifying AD-dementia from healthy individuals, and reached $91.54 \%$ when combined all regions ${ }^{[22]}$. By using hippocampal features, researchers can distinguish AD-dementia with an accuracy of $86.75 \%$, and $70.51 \%$ of $\mathrm{MCl}^{[19]}$. Identical conclusions were obtained in a recent large-scale multicenter study where the hippocampal features served as robust biomarkers for clinical identification of AD-dementia/MCl and further predicted whether $\mathrm{MCl}$ patients would convert to dementia ${ }^{[23]}$. By contrast, the deep learning methods indeed can acquire a slightly better diagnostic capabilities in Alzheimer's continuum ${ }^{[50]}$, however, it is difficult to explain the clinical correlations between these deep features and AD itself, and notably, $L i$ et al. have proved that the performance in identifying dementia from controls by using radiomics was comparable to deep learning (91.4\% and $93.9 \%$, respectively) ${ }^{[51]}$. Here, in distinguishing preclinical AD patients or clinical "converters", the accuracy of our models reached an amazing $81.86 \%-95.90 \%$, even higher than that of distinguishing symptomatic patients from controls. We thought several reasons may account for it. First, compared with extracting features solely on sMRI, we were based on MPMRI. Second, instead of selecting regions on prior knowledge, we adopted template segmentation and extracted features respectively. Third, among the huge 30128 features, we used an innovative selection method to improve the robustness. Four, we diagnosed individuals based on the $A \beta$ profile, not purely on clinical data, significantly reduced the heterogeneity of participants. Moreover, different types of models further verified the reliability.

The $A \beta$ deposition associated with neuronal degeneration may have resulted in subtle alterations in MRI signal intensity, therefore, we speculate that radiomics features can reflect changes at the microscopic level of early pathological status, which occurred before changes at the macroscopic level. In addition to the computer-aided classification, three stable high-frequency features, which were not affected by function perturbations (three different algorithms) and sample perturbations (5-fold cross validation and 100 repetitions), were identified during the selection process, they were the LZHGE feature of right posterior cingulate gyrus, Variance feature of left superior parietal gyrus and Coarseness feature of left posterior cingulate gyrus, all on sMRI modality. Importantly, the earliest accumulation of $A \beta$ deposition is also in the superior parietal gyrus and posterior cingulate ${ }^{[1]}$. More specifically, in symptomatic $A D$ patients, previous findings of autopsy and amyloid-PET suggested that parietal lobe and posterior cingulum are vulnerable to $A \beta$ invasion during early stage $[52,53]$; in cognitively normal individuals, the annual increase of $A \beta$ also localizes to these two regions ${ }^{[54]}$. From other perspectives, the $A \beta$ deposition is particularly associated with the cortical atrophy of superior parietal gyrus ${ }^{[55,56]}$, and the rate-limiting enzyme of $A \beta$ production is also significantly elevated in this area [57]. These developments prove the accuracy of locations, support our results that these features were correlated with SUVR values, played good roles in predicting future cognitive decline (AUCs $64.9 \%, 72.9 \%$ and $76.1 \%$ respectively; $83.9 \%$ when combined), and probably being the imaging biomarkers of preclinical AD. Interestingly, we found the retained features only came from sMRI modality, this is probably in part due to the relatively small number of fMRI 
and DTI features, additionally, a latest study concluded that DTI parameters is not useful for the identification of preclinical AD [58] and to the best of our knowledge, it is the first time that texture analysis of $\mathrm{fMRI}$ have been applied to the field of $A D{ }^{[36]}$. Uncertainty still exists, the significance of DTI and fMRI radiomics features cannot be completely denied in the exploratory study.

In the longitudinal analyses, we found levels of the Variance feature of left superior parietal gyrus on sMRI decreased with the impairment of cognition, suggesting it may of great importance in the whole cognitive continuum, not just in the preclinical stage. This feature is extracted from the gray-level cooccurrence matrix category, is an indicator of dispersion of the unit values around the mean. With cognition decline, the cortical accumulation of $A \beta$ will increase continuously in a certain extent ${ }^{[1]}$, may result in alterations in signal intensity, and then this subtle changes were captured by the radiomics analysis of sMRI. Next, we conducted survival analyses to compare the conversion time between groups within the cohort 3 . The median value was chosen subjectively for grouping; coincidently, we found also only the Variance feature can affect the conversion time, further suggested its predictive effects on clinical outcome.

Our study had some limitations. First, the small sample number limited the statistical power of our data. We tried to overcome this issue by enrolled participants from other sub-centers and ADNI, but the requirement of amyloid-PET or long-term follow-up or MPMRI greatly limited the quantity. Moreover, the performance of our models maybe different by using different imaging protocols. Second, considering no standard definition of "unstable preclinical $A D$ ", we referred to the 36 months of "unstable $\mathrm{MCl}$ ", and required the "non-converters" must remain cognitive stability at least three follow-up visits, while the average conversion time of "converters" was 41.2 months, which needs to be verified. Third, other regions, such as anterior cingulate, are also susceptible to A $\beta$ attack

$[52,53]$, however, we did not find any stable features situated in these regions. Fourth, in cohort 3 , most of the patients were limited at $\mathrm{MCl}$ stage and few of dementia stage at the follow-up timepoint, thus it is not clear whether features were related to the degree of cognitive deterioration. Fifth, the positive result of study 2 were not that significant $(p=0.0403)$, and the feature levels of some individuals were increased disparately, which probably due to the heterogeneity of $\mathrm{MCl}$ and the relatively older age of ADNI participants. Sixth, age may cause differences of our results because of its impacts on A $\beta$ and atrophy. Seventh, the positive rate of amyloid-PET (42.6\%) was higher than that of previous researches $\left(10 \%-30 \%\right.$ mostly) ${ }^{[59]}$, partly because of the exclusion of some negative individuals (Supplementary Figure 1A) and the existence of individuals with subjective cognitive decline, a high-risk state of developing AD [60]; this bias may increase uncertainty. Considering the shortcomings of our research and limitations in this field, multicenter collaboration to include more participants are needed in the further.

\section{Conclusions}

In conclusion, radiomics analysis of MPMRI is expected to become a new evaluation method for A $\beta$ deposition and future cognitive decline in cognitively healthy individuals, of great importance in diagnosing preclinical $A D$ and targeting super-early secondary prevention clinical trials. Additionally, we proposed a novel paradigm of features extraction and preservation method of features subset, solving the problem of instability and non-repeatability for future studies.

\section{Abbreviations}

AD: Alzheimer's disease; NIA-AA: National Institute on Ageing-Alzheimer's Association; Aß: amyloid- $\beta$; PET: positron emission tomography; MRI: Magnetic resonance imaging; fMRI: functional MRI; sMRI: structural MRI; MPMRI: multi-parameter MRI; DTI: diffusion tensor imaging; MCl: mild cognitive impairment; SILCODE: Sino Longitudinal Study on Cognitive Decline; ADNI: Alzheimer's Disease Neuroimaging Initiative; SUVR: standardized uptake value ratio; TR: repetition time; TE: echo time; EPI: echo planar imaging; ROI: region of interest; Lasso: Least absolute shrinkage and selection operator; mRMR: Max-Relevance and Min-Redundancy; SVM: support vector machine; RF: random forest; SD: standard deviation; ROC: receiver operating characteristic; AUCs: areas under curve; MoCA: Montreal cognitive assessment; LZHGE: Large zone high-gray-level emphasis.

\section{Declarations}

\section{Ethics approval and consent to participate}

The study was approved by the Medical Ethics Committee of Xuanwu Hospital of Capital Medical University and was carried out in accordance with the Declaration of Helsinki. We confirm that we have read the Journal's position on issues involved in ethical publication and affirm that this report is consistent with those guidelines.

\section{Consent for publication}

All subjects gave written informed consents and written consent to permit publication of clinical details.

\section{Availability of data and materials}

The datasets used and/or analysed during the current study are available from the corresponding author on reasonable request.

\section{Competing interests}

On behalf of all authors, the corresponding author confirms no conflict of interest. 


\section{Funding}

This work was supported by grants from the National Key Research and Development Program of China (2016YFC1306300, 2018YFC1312001), the National Natural Science Foundation of China (61633018).

\section{Authors' Contributions}

Ying $\mathrm{H}$ provided the data; JH Jiang designed the study; TR Li and Y Wu assembled and analyzed the data, consulted literatures and drafted the manuscript; J J Jiang drawn the Figure 1; Ying $\mathrm{H}$ and $\mathrm{JH}$ Jiang critically revised the manuscript for important intellectual content. All authors read and approved the final manuscript.

\section{Acknowledgments}

Data collection and sharing for this study was funded by the Sino Longitudinal Study on Cognitive Decline (SILCODE) project in China and the Alzheimer's Disease Neuroimaging Initiative (ADNl; National Institutes of Health Grant U19 AG024904), the authors wish to thank all the individuals who participated in the study and every staff member behind both projects.

\section{References}

1.Long JM, Holtzman DM. Alzheimer Disease: An Update on Pathobiology and Treatment Strategies. Cell. 2019. 179(2): 312-339.

2.Jia L, Quan M, Fu Y, et al. Dementia in China: epidemiology, clinical management, and research advances. Lancet Neurol. 2020. 19(1): 81-92.

3.Sperling RA, Jack CR Jr, Aisen PS. Testing the right target and right drug at the right stage. Sci Transl Med. 2011. 3(111): $111 \mathrm{~cm} 33$.

4.Golde TE, DeKosky ST, Galasko D. Alzheimer's disease: The right drug, the right time. Science. 2018. 362(6420): 1250-1251.

5.Jack CR Jr, Bennett DA, Blennow K, et al. NIA-AA Research Framework: Toward a biological definition of Alzheimer's disease. Alzheimers Dement. 2018. 14(4): 535-562.

6.Insel PS, Hansson O, Mackin RS, Weiner M, Mattsson N, Alzheimer's Disease Neuroimaging Initiative. Amyloid pathology in the progression to mild cognitive impairment. Neurobiol Aging. 2018. 64: 76-84.

7.Papp KV, Rentz DM, Mormino EC, et al. Cued memory decline in biomarker-defined preclinical Alzheimer disease. Neurology. 2017. 88(15): 1431-1438.

8.Li TR, Wang XN, Sheng C, et al. Extracellular vesicles as an emerging tool for the early detection of Alzheimer's disease. Mech Ageing Dev. 2019. 184: 111175 .

9.Promteangtrong C, Kolber M, Ramchandra P, et al. Multimodality Imaging Approach in Alzheimer disease. Part I: Structural MRI, Functional MRI, Diffusion Tensor Imaging and Magnetization Transfer Imaging. Dement Neuropsychol. 2015. 9(4): 318-329.

10.Rathore S, Habes M, Iftikhar MA, Shacklett A, Davatzikos C. A review on neuroimaging-based classification studies and associated feature extraction methods for Alzheimer's disease and its prodromal stages. Neuroimage. 2017. 155: 530-548.

11.Zhou H, Jiang J, Lu J, Wang M, Zhang H, Zuo C, et al. Dual-Model Radiomic Biomarkers Predict Development of Mild Cognitive Impairment Progression to Alzheimer's Disease. Front Neurosci. 2018;12:1045.

12.Li Y, Jiang J, Lu J, Jiang J, Zhang H, Zuo C. Radiomics: a novel feature extraction method for brain neuron degeneration disease using 18F-FDG PET imaging and its implementation for Alzheimer's disease and mild cognitive impairment. Ther Adv Neurol Disord. 2019;12:1756286419838682.

13.Guo Y, Zhang Z, Zhou B, et al. Grey-matter volume as a potential feature for the classification of Alzheimer's disease and mild cognitive impairment: an exploratory study. Neurosci Bull. 2014. 30(3): 477-89.

14.Baron JC, Chételat G, Desgranges B, et al. In vivo mapping of gray matter loss with voxel-based morphometry in mild Alzheimer's disease. Neuroimage. 2001. 14(2): 298-309.

15.Thomann PA, Wustenberg T, Pantel J, Essig M, Schroder J. Structural changes of the corpus callosum in mild cognitive impairment and Alzheimer's disease. Dement Geriatr Cogn Disord. 2006. 21(4): 215-20.

16.Pedro T, Weiler M, Yasuda CL, et al. Volumetric brain changes in thalamus, corpus callosum and medial temporal structures: mild Alzheimer's disease compared with amnestic mild cognitive impairment. Dement Geriatr Cogn Disord. 2012. 34(3-4): 149-55.

17.Balthazar ML, Yasuda CL, Pereira FR, Pedro T, Damasceno BP, Cendes F. Differences in grey and white matter atrophy in amnestic mild cognitive impairment and mild Alzheimer's disease. Eur J Neurol. 2009. 16(4): 468-74. 
18.Lee S, Lee H, Kim KW, Alzheimer's Disease Neuroimaging Initiative. Magnetic resonance imaging texture predicts progression to dementia due to Alzheimer disease earlier than hippocampal volume. J Psychiatry Neurosci. 2020. 45(1): 7-14.

19.Feng F, Wang P, Zhao K, et al. Radiomic Features of Hippocampal Subregions in Alzheimer's Disease and Amnestic Mild Cognitive Impairment. Front Aging Neurosci. 2018. 10: 290.

20.Sørensen L, Igel C, Liv Hansen N, et al. Early detection of Alzheimer's disease using MRI hippocampal texture. Hum Brain Mapp. 2016. 37(3): 1148-61.

21.Chaddad A, Desrosiers C, Niazi T. Deep Radiomic Analysis of MRI Related to Alzheimer's Disease. IEEE Access. 2018. 6: 58213-58221.

22.Chaddad A, Niazi T. Radiomics Analysis of Subcortical Brain Regions Related to Alzheimer Disease. IEEE Life Sci Conf. 2018.

23.Kun Zabc, Yanhui Dc, Ying Hdqrs, et al. Independent and reproducible hippocampal radiomic biomarkers for multisite Alzheimer's disease: diagnosis, longitudinal progress and biological basis. Sci Bull (Beijing). 2020. 65(13): 1103-1113.

24.Luk CC, Ishaque A, Khan M, et al. Alzheimer's disease: 3-Dimensional MRI texture for prediction of conversion from mild cognitive impairment. Alzheimers Dement (Amst). 2018. 10: 755-763.

25.Li S, Yuan X, Pu F, et al. Abnormal changes of multidimensional surface features using multivariate pattern classification in amnestic mild cognitive impairment patients. J Neurosci. 2014. 34(32): 10541-53.

26.de Oliveira MS, Balthazar ML, D'Abreu A, et al. MR imaging texture analysis of the corpus callosum and thalamus in amnestic mild cognitive impairment and mild Alzheimer disease. AJNR Am J Neuroradiol. 2011. 32(1): 60-6.

27.Feng Q, Chen Y, Liao Z, et al. Corpus Callosum Radiomics-Based Classification Model in Alzheimer's Disease: A Case-Control Study. Front Neurol. 2018. 9: 618.

28.Gyebnár G, Szabó Á, Sirály E, et al. What can DTI tell about early cognitive impairment? - Differentiation between MCl subtypes and healthy controls by diffusion tensor imaging. Psychiatry Res Neuroimaging. 2018. 272: 46-57.

29.Alves GS, O'Dwyer L, Jurcoane A, et al. Different patterns of white matter degeneration using multiple diffusion indices and volumetric data in mild cognitive impairment and Alzheimer patients. PLoS One. 2012. 7(12): e52859.

30.Li X, Wang X, Su L, Hu X, Han Y. Sino Longitudinal Study on Cognitive Decline (SILCODE): protocol for a Chinese longitudinal observational study to develop risk prediction models of conversion to mild cognitive impairment in individuals with subjective cognitive decline. BMJ Open. 2019. 9(7): e028188.

31.Chen G, Yang K, Du W, Hu X, Han Y (2019) Clinical Characteristics in Subjective Cognitive Decline with and without Worry: Baseline Investigation of the SILCODE Study. J Alzheimers Dis 72, 443-454.

32.McKhann GM, Knopman DS, Chertkow H, Hyman BT, Jack CR Jr, Kawas CH, Klunk WE, Koroshetz WJ, Manly JJ, Mayeux R, Mohs RC, Morris JC, Rossor MN, Scheltens P, Carrillo MC, Thies B, Weintraub S, Phelps CH (2011) The diagnosis of dementia due to Alzheimer's disease: recommendations from the National Institute on Aging-Alzheimer's Association workgroups on diagnostic guidelines for Alzheimer's disease. Alzheimers Dement 7, $263-269$.

33.Petersen RC (2004) Mild cognitive impairment as a diagnostic entity. J Intern Med 256, 183-194.

34.Bondi MW, Edmonds EC, Jak AJ, Clark LR, Delano-Wood L, McDonald CR, Nation DA, Libon DJ, Au R, Galasko D, Salmon DP (2014) Neuropsychological criteria for mild cognitive impairment improves diagnostic precision, biomarker associations, and progression rates. J Alzheimers Dis 42, $275-289$.

35.Fakhry-Darian D, Patel NH, Khan S, et al. Optimisation and usefulness of quantitative analysis of 18F-florbetapir PET. Br J Radiol. 2019. 92(1101): 20181020.

36.Hassan I, Kotrotsou A, Bakhtiari AS, et al. Radiomic Texture Analysis Mapping Predicts Areas of True Functional MRI Activity. Sci Rep. 2016.6 : 25295.

37.Tian Y, Liu Z, Tang Z, Li M, Lou X, Dong E, Liu G, Wang Y, Wang Y, Bian X, Wei S, Tian J, Ma L (2019) Radiomics Analysis of DTI Data to Assess Vision Outcome After Intravenous Methylprednisolone Therapy in Neuromyelitis Optic Neuritis. J Magn Reson Imaging 49, 1365-1373.

38.Tang Z, Liu Z, Li R, Yang X, Cui X, Wang S, et al. Identifying the white matter impairments among ART-naïve HIV patients: a multivariate pattern analysis of DTI data. Eur Radiol. 2017;27(10):4153-62.

39.Wang B, Liu Z, Liu J, Tang Z, Li H, Tian J. Gray and white matter alterations in early HIV-infected patients: Combined voxel-based morphometry and tractbased spatial statistics. J Magn Reson Imaging. 2016;43(6):1474-83.

40.Smith SM. Fast robust automated brain extraction. Hum Brain Mapp. 2002;17(3):143-55.

41.Basser PJ, Mattiello J, LeBihan D. MR diffusion tensor spectroscopy and imaging. Biophys J. 1994;66(1):259-67.

42.Chao-Gan Y, Yu-Feng Z. DPARSF: A MATLAB Toolbox for “Pipeline” Data Analysis of Resting-State fMRI. Front Syst Neurosci. $2010 ; 4: 13$. 
43.Smith SM, Jenkinson M, Woolrich MW, et al. Advances in functional and structural MR image analysis and implementation as FSL. Neuroimage. 2004. 23 Suppl 1: S208-19.

44.Vallières M, Freeman CR, Skamene SR, El Naqa I. A radiomics model from joint FDG-PET and MRI texture features for the prediction of lung metastases in soft-tissue sarcomas of the extremities. Phys Med Biol. 2015. 60(14): 5471-96.

45.Cui Z, Zhong S, Xu P, He Y, Gong G. PANDA: a pipeline toolbox for analyzing brain diffusion images. Front Hum Neurosci. $2013.7: 42$.

46.Zhao W, Wang X, Yin C, He M, Li S, Han Y. Trajectories of the Hippocampal Subfields Atrophy in the Alzheimer's Disease: A Structural Imaging Study. Front Neuroinform. 2019. 13: 13.

47.Li XY, Tang ZC, Sun Y, Tian J, Liu ZY, Han Y. White matter degeneration in subjective cognitive decline: a diffusion tensor imaging study. Oncotarget. 2016. 7(34): 54405-54414.

48.Shu N, Wang X, Bi Q, Zhao T, Han Y. Disrupted Topologic Efficiency of White Matter Structural Connectome in Individuals with Subjective Cognitive Decline. Radiology. 2018. 286(1): 229-238.

49.Yan T, Wang W, Yang L, Chen K, Chen R, Han Y. Rich club disturbances of the human connectome from subjective cognitive decline to Alzheimer's disease. Theranostics. 2018. 8(12): 3237-3255.

50.Jo T, Nho K, Saykin AJ. Deep Learning in Alzheimer's Disease: Diagnostic Classification and Prognostic Prediction Using Neuroimaging Data. Front Aging Neurosci. 2019. 11: 220.

51.Li H, Habes M, Yong F (2017) Deep Ordinal Ranking for Multi-Category Diagnosis of Alzheimer's Disease using Hippocampal MRI data. arXiv: Computer Vision and Pattern Recognition

52.Thal DR, Rüb U, Orantes M, Braak H. Phases of A beta-deposition in the human brain and its relevance for the development of AD. Neurology. 2002. 58(12): $1791-800$.

53.Cho H, Lee HS, Choi JY, et al. Predicted sequence of cortical tau and amyloid- $\beta$ deposition in Alzheimer disease spectrum. Neurobiol Aging. 2018. 68: 7684 .

54.Sojkova J, Zhou Y, An Y, et al. Longitudinal patterns of $\beta$-amyloid deposition in nondemented older adults. Arch Neurol. 2011. 68(5): 644-9.

55.Becker JA, Hedden T, Carmasin J, et al. Amyloid- $\beta$ associated cortical thinning in clinically normal elderly. Ann Neurol. 2011. 69(6): 1032-42.

56.Weston PS, Nicholas JM, Lehmann M, et al. Presymptomatic cortical thinning in familial Alzheimer disease: A longitudinal MRI study. Neurology. 2016. 87(19): 2050-2057.

57.Coulson DT, Beyer N, Quinn JG, et al. BACE1 mRNA expression in Alzheimer's disease postmortem brain tissue. J Alzheimers Dis. 2010. $22(4)$ : 1111-22.

58.Teipel SJ, Kuper-Smith JO, Bartels C, et al. Multicenter Tract-Based Analysis of Microstructural Lesions within the Alzheimer's Disease Spectrum: Association with Amyloid Pathology and Diagnostic Usefulness. J Alzheimers Dis. 2019. 72(2): 455-465.

59.Chételat G, La Joie R, Villain N, Perrotin A, de La Sayette V, Eustache F, Vandenberghe R (2013) Amyloid imaging in cognitively normal individuals, at-risk populations and preclinical Alzheimer's disease. Neuroimage Clin 2, 356-365.

60.Jessen F, Amariglio RE, Buckley RF, van der Flier WM, Han Y, Molinuevo JL, Rabin L, Rentz DM, Rodriguez-Gomez O, Saykin AJ, Sikkes S, Smart CM, Wolfsgruber S, Wagner M (2020) The characterisation of subjective cognitive decline. Lancet Neurol 19, 271-278.

\section{Tables}




\begin{tabular}{|c|c|c|c|c|c|c|c|c|}
\hline & Cohort 1 & & & Cohort 2 & & & & Cohort 3 \\
\hline Group & $A \beta \_P(n=78)$ & $\begin{array}{l}A \beta \_N(n= \\
105)\end{array}$ & $P$ value & Cog_D $(n=24)$ & Cog_M $(n=27)$ & $\begin{array}{l}P \\
\text { value }\end{array}$ & ADNI $(n=13)$ & $\begin{array}{l}\text { ADNI + Cog_D }(n= \\
37)\end{array}$ \\
\hline $\begin{array}{l}\text { Gender } \\
(\mathrm{M} / \mathrm{F})\end{array}$ & $21 / 57$ & $36 / 69$ & 0.280 & $11 / 13$ & $11 / 16$ & 0.714 & $4 / 9$ & $15 / 22$ \\
\hline Age (y) & $67.4 \pm 6.0$ & $65.6 \pm 5.5$ & $0.039^{*}$ & $69.5 \pm 7.6$ & $68.9 \pm 7.3$ & 0.775 & $74.85 \pm 6.75$ & $73.32 \pm 9.06$ \\
\hline Education & $12.8 \pm 3.6$ & $12.3 \pm 3.1$ & 0.275 & $11.0 \pm 4.8$ & $13.0 \pm 2.7$ & 0.074 & $14.7 \pm 1.2$ & $12.30 \pm 4.29$ \\
\hline MoCA & $26.2 \pm 3.1$ & $25.9 \pm 2.8$ & 0.486 & $\begin{array}{l}23.5 \pm 1.9 \\
(23 \text { Ava) }\end{array}$ & $\begin{array}{l}25.0 \pm 3.2 \\
(26 \text { Ava) }\end{array}$ & 0.054 & $\begin{array}{l}23.8 \pm 3.8 \\
(12 A v a)\end{array}$ & $23.7 \pm 2.7$ (35Ava) \\
\hline APOE $\varepsilon 4$ & $\begin{array}{l}28 \text { (77Ava, } \\
36.4 \%)\end{array}$ & $38(36.2 \%)$ & 0.981 & $\begin{array}{l}9(21 \mathrm{Ava}, \\
42.9 \%)\end{array}$ & $13(48.1 \%)$ & 0.715 & $1(7.7 \%)$ & 10 (34Ava, 29.4\%) \\
\hline SUVR & $1.230 \pm 0.047$ & $1.089 \pm 0.057$ & $<0.0001^{\star *}$ & / & / & / & / & / \\
\hline
\end{tabular}

Table 1. Clinical characteristics of participants.

The cohort 1 was qualitatively divided into A $\beta-P$ and A $-N$ groups according to participants' SUVR (cutoff of 1.18); the cohort 2 was classified by participants' future cognitive outcomes, including Cog-D and Cog-M groups. At baseline timepoint, all participants were cognitively healthy, and we made comparisons of clinical data between the two groups of cohort 1 and 2, respectively. The cohort 1 was used as the training and validation dataset, the cohort 2 was used as the test set. The cohort 3 was applied to the longitudinal study, including the 24 participants from cohort 2 (Cog-D) and 13 from ADNI, the latter was a supplement and identical to the evaluation of Cog-D group. The MoCA scale applied in cohort 1 was Chinese MoCA-Basic version, in cohort 2 was MoCABeijing version, and in ADNI was traditional MoCA version. Continuous measures were presented as mean \pm standard deviation. Statistical analysis was conducted by Chi-square test for categorical variables and independent two-sample two-tailed t test for quantitative variables. * means $p<0.05$; ** means $p<0.001$.

Abbreviations: $A \beta$, amyloid- $\beta$; P, positive; N, negative; Cog, cognition; D, deteriorated; $M$, maintained; $M$, male; $F$, female; MoCA, Montreal Cognitive Assessment; SUVR, standard uptake value ratio; ADNI, Alzheimer's Disease Neuroimaging Initiative; Ava, available.

\begin{tabular}{|c|c|c|c|c|c|c|c|c|c|c|}
\hline \multicolumn{4}{|c|}{$\begin{array}{l}\text { Two-sample t-test, autocorrelation and Fisher } \\
\text { score }\end{array}$} & \multicolumn{4}{|c|}{ Two-sample t-test, autocorrelation and Lasso } & \multicolumn{3}{|c|}{ Two-sample t-test, autocorrelation and $\mathrm{r}$} \\
\hline $\begin{array}{l}\text { Feature } \\
\text { (ID) }\end{array}$ & Times & Brain region & $\mathbf{R}$ & Feature (ID) & Times & Brain region & $\mathbf{R}$ & $\begin{array}{l}\text { Feature } \\
\text { (ID) }\end{array}$ & Times & Brain region \\
\hline $\begin{array}{l}\text { LZHGE } \\
(6486)\end{array}$ & 500 & Cingulum_Post_L & $1 / 2$ & $\begin{array}{l}\text { Busyness } \\
(26056)\end{array}$ & 468 & Frontal_Mid_Orb_R & 2 & $\begin{array}{l}\text { LZHGE } \\
(6486)\end{array}$ & 495 & Cingulum_Post \\
\hline $\begin{array}{l}\text { LZHGE } \\
(6529)\end{array}$ & 500 & Cingulum_Post_R & $1 / 2$ & $\begin{array}{l}\text { Homogeneity } \\
(24775)\end{array}$ & 467 & Vermis_7 & $3 / 2$ & $\begin{array}{l}\text { LZHGE } \\
(6529)\end{array}$ & 488 & Cingulum_Post \\
\hline $\begin{array}{l}\text { LZHGE } \\
(11474)\end{array}$ & 500 & Cingulum_Post_L & $2 / 3$ & $\begin{array}{l}\text { Variance } \\
(27442)\end{array}$ & 430 & Parietal_Sup_L & 2 & $\begin{array}{l}\text { LZHGE } \\
(\mathbf{1 1 5 1 7})\end{array}$ & 486 & Cingulum_Post \\
\hline $\begin{array}{l}\text { LZHGE } \\
(11517)\end{array}$ & 500 & Cingulum_Post_R & $2 / 3$ & $\begin{array}{l}\text { Contrast } \\
(14273)\end{array}$ & 419 & Cerebelum_6_R & $2 / 3$ & $\begin{array}{l}\text { ZSN } \\
(27076)\end{array}$ & 445 & Occipital_Sup_ \\
\hline $\begin{array}{l}\text { Variance } \\
(\mathbf{2 7 4 4 2 )}\end{array}$ & 480 & Parietal_Sup_L & 2 & $\begin{array}{l}\text { Complexity } \\
\text { (9287) }\end{array}$ & 402 & Cerebelum_6_R & $1 / 2$ & $\begin{array}{l}\text { LZHGE } \\
(11474)\end{array}$ & 441 & Cingulum_Post \\
\hline $\begin{array}{l}\text { LZLGE } \\
(24803)\end{array}$ & 447 & Vermis_7 & $3 / 2$ & $\begin{array}{l}\text { Coarseness } \\
(\mathbf{6 4 8 9})\end{array}$ & 399 & Cingulum_Post_L & $1 / 2$ & $\begin{array}{l}\text { Variance } \\
\text { (27442) }\end{array}$ & 441 & Parietal_Sup_L \\
\hline $\begin{array}{l}\text { Strength } \\
(18834)\end{array}$ & 428 & Temporal_Inf_R & 1 & $\begin{array}{l}\text { Kurtosis } \\
(7485)\end{array}$ & 397 & Parietal_Sup_L & $1 / 2$ & $\begin{array}{l}\text { SZLGE } \\
(11471)\end{array}$ & 420 & Cingulum_Post \\
\hline $\begin{array}{l}\text { Coarseness } \\
(6489)\end{array}$ & 423 & Cingulum_Post_L & $1 / 2$ & $\begin{array}{l}\text { Busyness } \\
(26314)\end{array}$ & 394 & Cingulum_Ant_R & 2 & $\begin{array}{l}\text { SZLGE } \\
(18179)\end{array}$ & 398 & Temporal_Mild \\
\hline $\begin{array}{l}\text { ZSN } \\
(27076)\end{array}$ & 423 & Occipital_Sup_R & 2 & $\begin{array}{l}\text { LZHGE } \\
(\mathbf{1 1 5 1 7})\end{array}$ & 391 & Cingulum_Post_R & $2 / 3$ & $\begin{array}{l}\text { Coarseness } \\
(\mathbf{6 4 8 9 )}\end{array}$ & 339 & Cingulum_Post \\
\hline $\begin{array}{l}\text { GLN } \\
(16497)\end{array}$ & 420 & Cingulum_Post_R & 1 & $\begin{array}{l}\text { Kurtosis } \\
(5292)\end{array}$ & 383 & Frontal_Mid_R & $1 / 2$ & $\begin{array}{l}\text { ZSV } \\
\text { (28977) }\end{array}$ & 320 & Cerebelum_Cru \\
\hline
\end{tabular}

Table 2. The high-frequency features selected by cross-validation with different methods. 
Under the sample disturbance of 5-fold cross-validation, we have carried on three different kinds of composite function disturbances separately to screen features in the training dataset, and repeated the process 100 times. We calculated the number of occurrences of each retained feature, ranging from 0 to 500 , and listed the top ten most frequently appeared features here, they were all originated from sMRI modality. Three stable high-frequency features were verified, and their identify numbers were 11517, 27442 and 6489 respectively. The Kurtosis feature belong to the "global" category; the Homogeneity and Variance features belong to the "gray-level co-occurrence matrix" category; the GLN, ZSN, LZHGE, SZLGE, LZLGE and ZSV features belong to the "gray-level size zone matrix" category; the Strength, Coarseness, Busyness, Complexity and Contrast features belong to the "neighborhood gray-tone difference matrix" category. Notably, the Variance and Contrast feature can also originate from the "global" and "gray-level co-occurrence matrix" category, respectively.

Abbreviations: Lasso, Least absolute shrinkage and selection operator; mRMR, Max-Relevance and Min-Redundancy; ID, identify number; sMRI, structural magnetic resonance imaging; L, left; R, right; Post, posterior; Sup, superior; Inf, inferior; Mid, middle; Orb, orbital; Ant, anterior; GLN, Gray-level nonuniformity; ZSN, Zone-size nonuniformity; LZHGE, Large zone high-gray-level emphasis; SZLGE, Small zone low gray-level emphasis; LZLGE, Large zone low-gray-level emphasis; ZSV, Zone-size variance.

\begin{tabular}{|c|c|c|c|c|c|c|c|}
\hline \multirow[b]{2}{*}{ Group } & \multirow[b]{2}{*}{ Method } & \multicolumn{3}{|c|}{ SVM model (radial basis kemel) } & \multicolumn{3}{|l|}{ RF model } \\
\hline & & ACC & SEN & SPE & ACC & SEN & SPE \\
\hline Validation dataset & Fisher score & $90.23 \% \pm 4.78 \%$ & $85.91 \% \pm 8.94 \%$ & $93.71 \% \pm 5.45 \%$ & $87.07 \% \pm 5.53 \%$ & $80.29 \% \pm 10.53 \%$ & $92.46 \% \pm 5.85 \%$ \\
\hline Test dataset & Fisher score & $84.48 \% \pm 5.58 \%$ & $82.87 \% \pm 12.56 \%$ & $85.98 \% \pm 7.11 \%$ & $83.19 \% \pm 5.89 \%$ & $77.80 \% \pm 12.38 \%$ & $88.19 \% \pm 6.47 \%$ \\
\hline Validation dataset & Lasso & $95.90 \% \pm 3.29 \%$ & $92.82 \% \pm 6.84 \%$ & $98.26 \% \pm 2.80 \%$ & $90.81 \% \pm 4.76 \%$ & $84.58 \% \pm 9.13 \%$ & $95.74 \% \pm 4.60 \%$ \\
\hline Test dataset & Lasso & $88.94 \% \pm 5.33 \%$ & $80.56 \% \pm 10.71 \%$ & $96.70 \% \pm 3.83 \%$ & $83.68 \% \pm 6.85 \%$ & $74.26 \% \pm 13.86 \%$ & $92.41 \% \pm 5.78 \%$ \\
\hline Validation dataset & mRMR & $93.00 \% \pm 4.19 \%$ & $89.07 \% \pm 7.11 \%$ & $96.08 \% \pm 3.98 \%$ & $90.28 \% \pm 4.97 \%$ & $84.14 \% \pm 10.03 \%$ & $95.08 \% \pm 4.96 \%$ \\
\hline Test dataset & mRMR & $86.08 \% \pm 5.68 \%$ & $79.76 \% \pm 12.13 \%$ & $91.93 \% \pm 5.00 \%$ & $83.52 \% \pm 6.62 \%$ & $75.85 \% \pm 13.47 \%$ & $90.63 \% \pm 6.01 \%$ \\
\hline
\end{tabular}

Table 3. Classification performance of the SVM model (radial basis kernel) and RF model.

Under the sample disturbance of 5-fold cross-validation, we have carried on three different kinds of composite function disturbances separately to screen features in the training dataset, and repeated the process 100 times. The retained features were incorporated into the SVM model and RF model each time, then we calculated the models' classification performance in the validation dataset and test dataset separately. The measures were presented as mean \pm standard deviation.

Abbreviations: SVM, support vector machine; RF, random forest; ACC, accuracy; SEN, sensibility; SPE, specificity; Lasso, Least absolute shrinkage and selection operator; mRMR, Max-Relevance and Min-Redundancy.

\section{Figures}

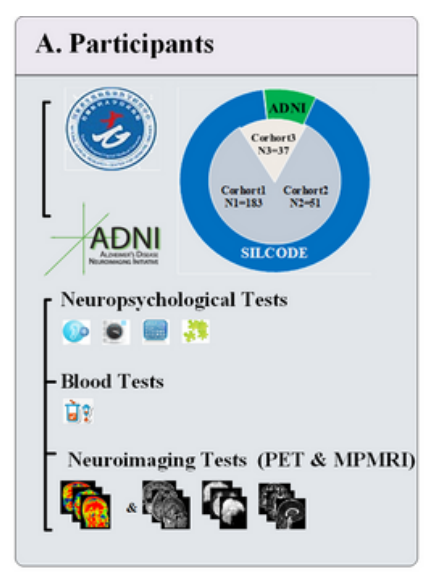

\section{B. Preprocessing}

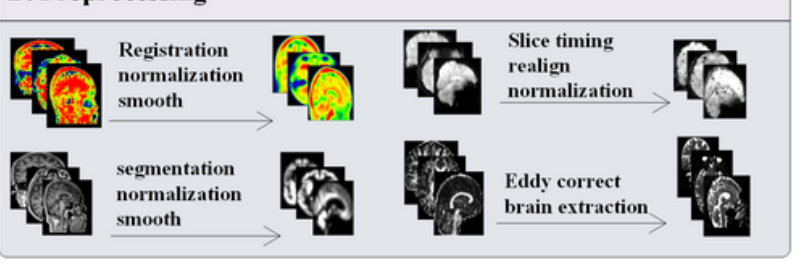

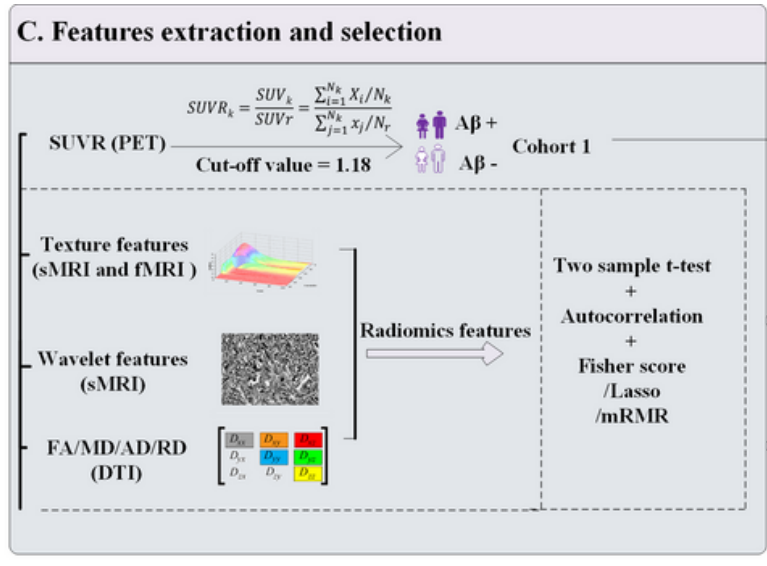

\section{Classification and correlation}

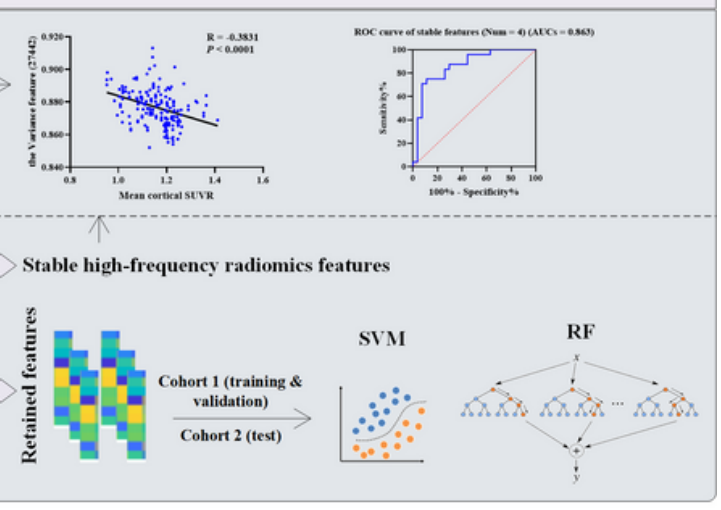

\section{E. Longitudinal analysis}

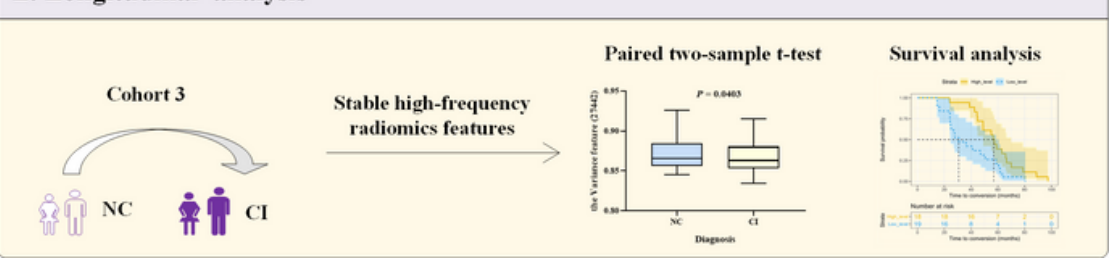


Figure 1

Workflow diagram. A, three cohorts were enrolled. The cohort $1(n=183)$ and $2(n=51)$ were both from SILCODE project, and divided qualitatively by A $\beta$ status or future cognitive outcomes, respectively; the cohort $3(n=37)$ included 24 "converters" from SILCODE and 13 from ADNI. All participants were cognitively healthy at baseline timepoint and were evaluated in a standardized protocol. B, preprocessing of amyloid-PET and MPMRI was performed for each modality respectively. C, radiomics features were extracted from each modality respectively; a novel method characterized by the combination of function perturbations (t-test, autocorrelation test, and three independent algorithms) and sample perturbations (5-fold cross-validation and 100 repetitions) was performed to select features from the training dataset ( $80 \%$ of cohort 1$)$. D, retained features were incorporated into classification models and verified in the validation (the rest $20 \%$ of cohort 1 ) and test dataset (cohort 2), respectively. Furthermore, during the process of selection, stable high-frequency features were identified, they were undisturbed by perturbations and correlated with the SUVR values, played good roles in predicting prospective cognitive decline (cohort 2). E, levels of stable high-frequency features were tested whether change with cognitive decline or impact the conversion time. Abbreviations: ADNI, Alzheimer's Disease Neuroimaging Initiative; MRI, magnetic resonance imaging; sMRI, structural MRI; fMRI, functional MRI; DTI, diffusion tensor imaging; MPMRI, multi-parameter MRI; PET, positron emission tomography; FA, fractional anisotropy; MD, mean diffusivity; AD, axial diffusivity; RD, radial diffusivity; SUVR, standard uptake value ratio; $A \beta$, amyloid $\beta$; Lasso, Least absolute shrinkage and selection operator; mRMR, Max-Relevance and Min-Redundancy; SVM, support vector machine; $\mathrm{RF}$, random forest; ROC, receiver operating characteristic; $\mathrm{AUCs}$, areas under curve; $\mathrm{NC}$, normal control; $\mathrm{Cl}$, cognitive impairment.

A

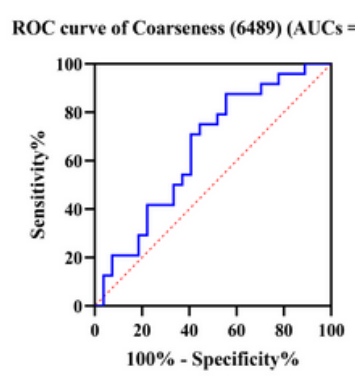

E

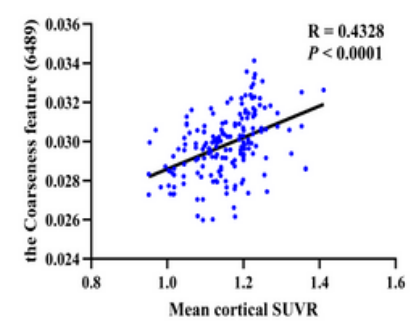

B

ROC curve of LZHGE (11517) (AUCs = 0.729)

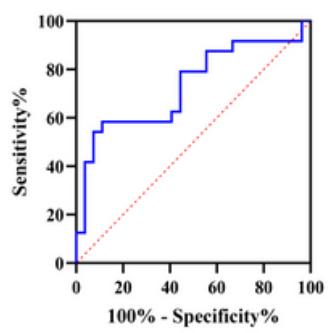

$\mathbf{F}$

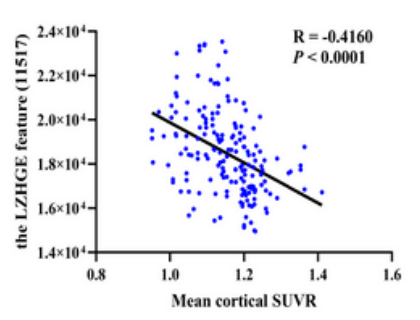

C

ROC curve of Variance (27442) (AUCs $=0.761)$

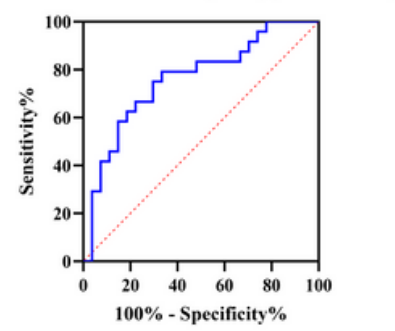

G

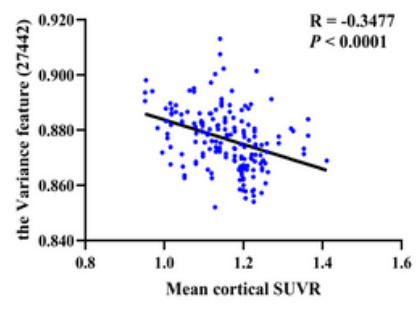

D

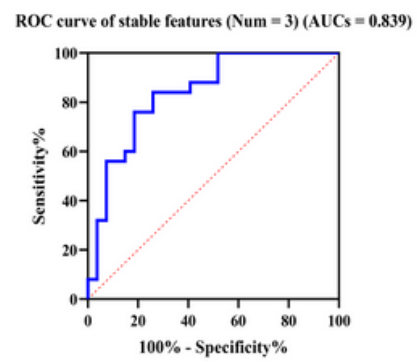

Figure 2

The ROC curves of stable high-frequency features and correlation analysis. A-D showed the ROC curves of stable high-frequency features in the test dataset, they all have high discriminating power. In detail, the 6489 feature, AUCs $=0.649(A)$, the 11517 feature, AUCs $=0.729(B)$, and the 27442 feature, AUCs $=0.761$ (C). The value increased to 0.839 when combined them together (D). E-G showed the correlations of levels of these features and mean cortical SUVR values in participants of cohort 1. The features were the Coarseness feature of left posterior cingulate gyrus on sMRI (ID: 6489) (A), the LZHGE feature of right posterior cingulate gyrus on sMRI (ID: 11517) (B) and the Variance feature of left superior parietal gyrus on sMRI (ID: 27442) (C). Abbreviations: LZHGE, Large zone high-gray-level emphasis; SUVR, standardized uptake value ratio; ROC, receiver operating characteristic; AUCs, areas under curve; sMRI, structural magnetic resonance imaging; Num, number. 
A

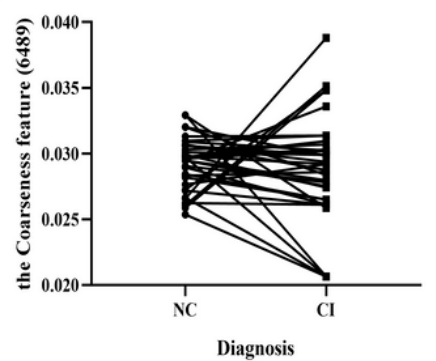

D

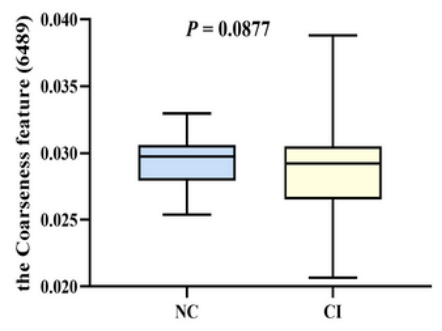

G
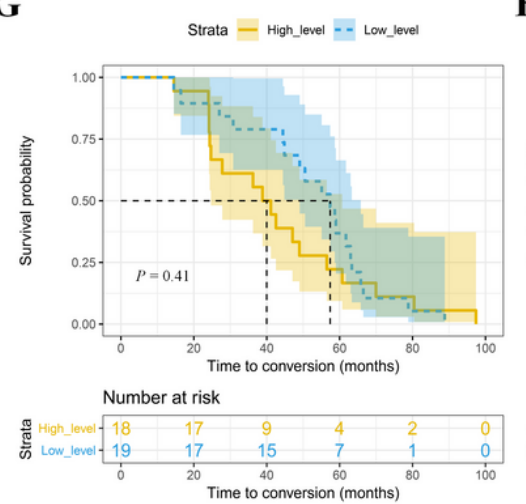

B

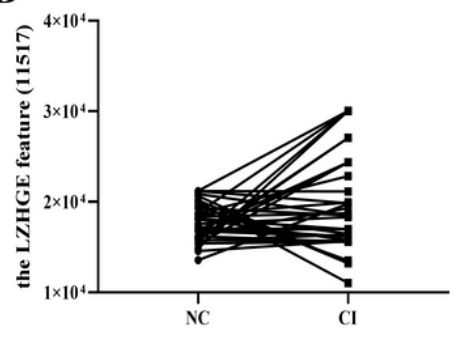

Diagnosis

E

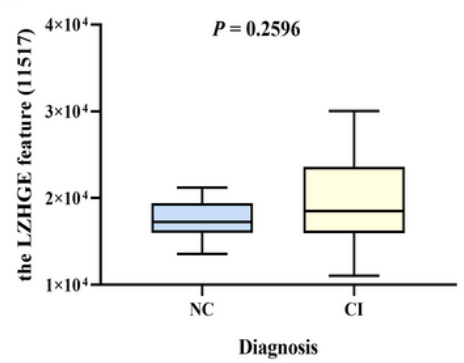

H

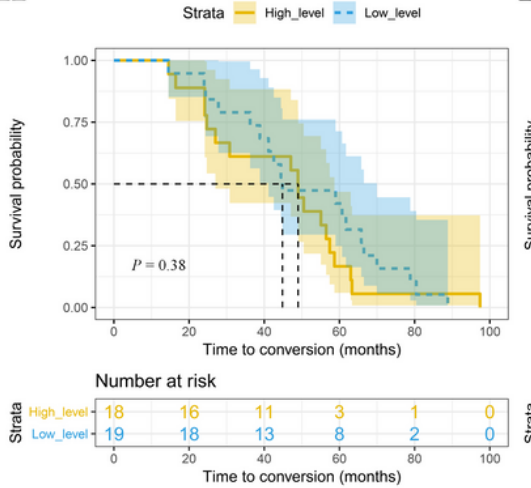

C

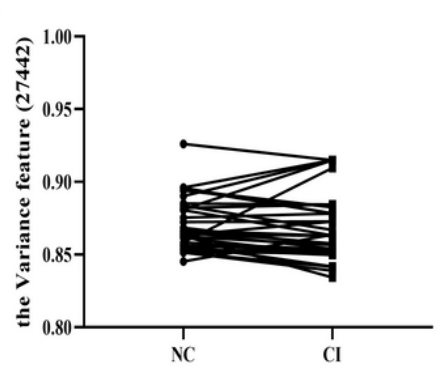

$\mathbf{F}$

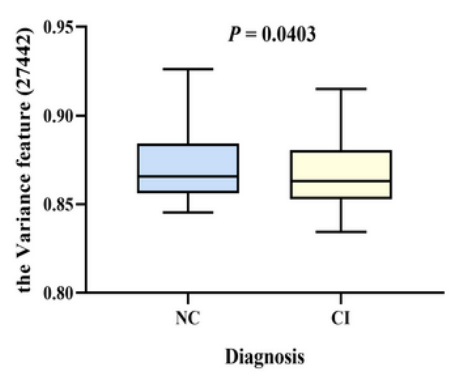

I

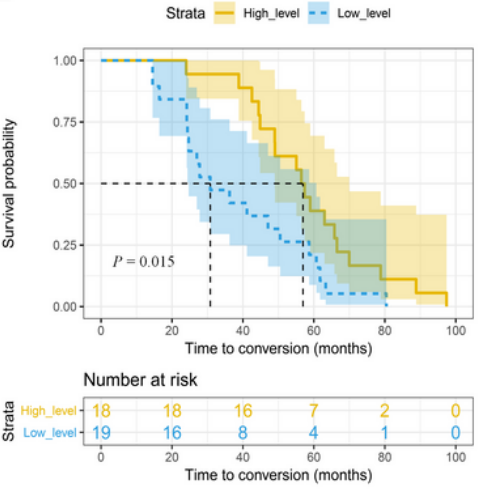

\section{Figure 3}

The longitudinal changes and survival analyses of stable high-frequency features. In study 2, 37 participants were included, their cognition was normal at baseline while impaired during follow-up, with 2 progressed to dementia and 35 to mild cognitive impairment. We made comparisons of levels of each stable high-frequency feature between the two timepoints; A-C showed the changing trajectory at the individual level; D-F were at the group level, with paired twosample t-test (two-tailed, $p<0.05)$. Furthermore, these individuals were stratified into high-level group $(n=18)$ and low-level group ( $n=19)$ by the baseline median level of each stable high-frequency feature, respectively; G-I were Kaplan-Meier curves showed the cumulative probabilities of conversion of the two groups (shaded area was the $95 \%$ confidence interval); difference was displayed by making log-rank tests $(p<0.05)$. Only the feature 27442 was different at the two timepoints $(p=0.0403)$, and its low-level group has a shorter conversion time than the high-level group $(p=0.015)$. The features were the Coarseness feature of left posterior cingulate gyrus on sMRI (ID: 6489) (A, D, G), the LZHGE feature of right posterior cingulate gyrus on sMRI (ID: 11517) (B, E, H), and the Variance feature of left superior parietal gyrus on SMRI (ID: 27442) (C, F, I). Abbreviations: NC, normal control; Cl, cognitive impairment; sMRI, structural magnetic resonance imaging; LZHGE, Large zone high-gray-level emphasis.

\section{Supplementary Files}

This is a list of supplementary files associated with this preprint. Click to download.

- 2020.07.27SupplementaryMaterialfinal.docx

- 2020.07.27SupplementaryFigure2.tif

- 2020.07.27SupplementaryFigure3.tif

- 2020.07.27SupplementaryFigure1.tif

- 2020.07.27SupplementaryFigure4.tif 